\title{
Article
}

\section{The Observed Linearity and Detection Response of Magnetic Fluid Concentration Magnetometry - A Theoretical and Experimental Description}

Mercer, Tim and Bissell, Philip

Available at http://clok.uclan.ac.uk/7752/

Mercer, Tim ORCID: 0000-0002-1557-2138 and Bissell, Philip ORCID: 00000002-8024-1757 (2013) The Observed Linearity and Detection Response of Magnetic Fluid Concentration Magnetometry - A Theoretical and Experimental Description. IEEE Transactions on Magnetics, 49 (7). pp. 3516-3519. ISSN 0018-9464

It is advisable to refer to the publisher's version if you intend to cite from the work. http://dx.doi.org/10.1109/TMAG.2013.2245867

For more information about UCLan's research in this area go to http://www.uclan.ac.uk/researchgroups/ and search for <name of research Group>.

For information about Research generally at UCLan please go to http://www.uclan.ac.uk/research/

All outputs in CLoK are protected by Intellectual Property Rights law, including Copyright law. Copyright, IPR and Moral Rights for the works on this site are retained by the individual authors and/or other copyright owners. Terms and conditions for use of this material are defined in the policies page. 
(C) 2013 IEEE. Personal use of this material is permitted. Permission from IEEE must be obtained for all other users, including reprinting/ republishing this material for advertising or promotional purposes, creating new collective wథrks for resale or redistribution to servers or lists, or reuse of any copyrighted components of this work in other works.

\title{
The Observed Linearity and Detection Response of Magnetic Fluid Concentration Magnetometry - A Theoretical and Experimental Description
}

\author{
Tim Mercer ${ }^{1,2,3}$, and Philip R. Bissell ${ }^{1,2}$ \\ ${ }^{1}$ Jeremiah Horrocks Institute for Mathematics, Physics \& Astronomy, University of Central Lancashire, Preston, PR1 2HE, UK \\ ${ }^{2}$ Centre for Materials Science, University of Central Lancashire, Preston, PR1 2HE, UK \\ ${ }^{3}$ Department of Physics, University of Liverpool, Liverpool, L69 7ZE, UK
}

\begin{abstract}
The response of a Scanning Column Magnetometer (SCM) used to measure concentration profiles of columns of magnetic dispersions has been investigated experimentally and theoretically. From the observed linearity of the total SCM output signal as a function of magnetic particle concentration, a consistent theoretical description is developed that assumes a small sensing coil field and no on-going particle agglomeration. Further theoretical development resulted in a detection coil response function that compared well with the measured response of a delta function approximation in the form of a thin ferrite disk and means that the SCM response function and spatial resolution may be determined from the coil design alone.
\end{abstract}

Index Terms - Instrument Response Function, Magnetic Fluids, Magnetic Particle Concentration, Scanning Column Magnetometry,

\section{INTRODUCTION}

$\mathrm{T}$ HE FABRICATION and characterization of dispersions of magnetic particles continues to be an important field of study with a wide range of applications that includes the wellestablished ferrofluids and their association with industrial/engineering uses [1], through to suspensions used in the fabrication of high-end flexible data storage media [2] and to the increasing interest in dispersions of superparamagnetic iron oxide nanoparticles for potential biomedical applications such as those of drug delivery, contrasting agents and magnetic hyperthermia [3].

For the measurement of magnetic fluid concentration, the change in inductance of a detection coil is a well-known and convenient technique. By scanning a column of dispersion with the coil, a complete concentration profile of the fluid as a function of height may be obtained. This provides the opportunity to go beyond simple stability studies and extend into investigations of the aggregation of particles into structural units that also contain trapped fluid [4].

The experimental method relies upon the observed linearity of the instrument output signal as a function of magnetic particle concentration. By investigating the origin of this linearity, a complete theoretical description of the detection response is developed here for the first time and is used to determine the detection response of a Scanning Column Magnetometer (SCM) from the coil design alone. Confidence in the result was obtained by comparison with the measured experimental response of an SCM of the same coil design.

\section{OBSERVED LINEARITY OF THE SCANNING COLUMN} MAGNETOMETER

\section{A. Principle of Operation}

The SCM at UCLan was built in-house, with a schematic of the instrument shown in Fig. 1. Here, a tube of magnetic dispersion is driven down by a computer controlled stepper motor through the otherwise empty core of the detection coil.

\section{FIG. 1 HERE}

As the coil forms the inductive part of a Colpitts oscillator resonance circuit, the change in the inductance of the coil (as magnetic material is introduced into its core) causes a corresponding change in oscillator frequency, $\Delta F$, from that of the sample-free frequency of $1 \mathrm{MHz}$. This change is recorded as the output signal of the SCM via the computer interface and hence a plot of $\Delta F$ as a function of suspension column height yields a complete concentration profile of the fluid.

\section{B. Output Signal of the SCM as a Function of Magnetic Pigment Concentration: Experimental Details and Results}

In order to investigate the output, $\Delta F$, as a function of magnetic Pigment Volume Concentration ( $P V C$ - also known as the volumetric packing fraction), a dispersion based on a well-known commercial formulation of $\gamma-\mathrm{Fe}_{2} \mathrm{O}_{3}$ particles used to manufacture a 300 Oe data/archive tape was produced inhouse using standard bead milling and letdown techniques. This system was chosen as our model for two important reasons: (i) the formulation is known to be highly stable, yielding well dispersed particles with little agglomeration and (ii) $\gamma-\mathrm{Fe}_{2} \mathrm{O}_{3}$ can be readily transformed by simple heat treatment into its antiferromagnetic allotrope $\alpha-\mathrm{Fe}_{2} \mathrm{O}_{3}$ providing the means to effectively turn off the magnetic moments of the particles whilst maintaining their chemical properties. In this way dispersions could be produced with a proportion of the particles replaced by their nonmagnetic equivalent in order to dilute the magnetic content-only of the suspension. Whilst full details of the formulation are proprietary, it can be disclosed that particles were of given mean length $300 \mathrm{~nm}$ and aspect ratio 5:1 suspended in an organic solvent-resin solution.

Heating of the $\gamma-\mathrm{Fe}_{2} \mathrm{O}_{3}$ pigment was carried out in a carbolite furnace at about $550{ }^{\circ} \mathrm{C}$. Samples were removed at regular intervals and the magnetization, $M$, measured using a standard vibrating sample magnetometer. After 78 hours the 
reduction in $M$ had flattened off to $<1.2 \%$ of the original gamma value of $75 \mathrm{emu} / \mathrm{g}$ giving an estimate of final alpha purity of $>98.8 \%$. At temperatures above $400{ }^{\circ} \mathrm{C}$ this conversion is expected as there is enough energy to cause the small change in atomic positions needed to directly transform the metastable gamma phase of the iron oxide (brown maghemite) from a cubic structure to the preferred (lower energy) rhombohedral structure of the alpha phase (red hematite) [5]. In addition to the magnetic measurements and change in color from brown to red, X-ray diffraction results using Cobalt $K_{\alpha}$ radiation matched well with those of the standard JCPDS file no. 24-72 [6] to confirm $\alpha-\mathrm{Fe}_{2} \mathrm{O}_{3}$ as the phase formed. Scanning Electron Micrographs also indicated that the heating process had not caused any sintering of the particles or changes to size and shape, thereby ensuring that the only change in mixed gamma-alpha dispersions would be that of magnetic particle concentration alone.

A series of dispersions were produced that varied the magnetic pigment content whilst keeping the total PVC constant at $5.5 \%$ using the nonmagnetic phase. The resultant frequency shift in the SCM signal is shown in Fig. 2 as a function of the normalized magnetic PVC/total PVC.

\section{FIG. 2 HERE}

Each data point in the main plot was calculated from the mean step height of the SCM concentration profiles at time zero (immediately after the final milling process and prior to any sedimentation). The profile for the sample at full magnetic content is shown in the inset with the mean taken between points $a$ and $a^{\prime}$. For this test to be valid a high quality (well dispersed) homogenous suspension is required as indicated by the near top hat shape of the profile. The concentration is almost constant over the dispersion height with the standard error between $a$ and $a^{\prime}$ at less than $0.02 \%$ of the mean. In fact, the errors for all the data points of the main plot were found to be $<0.04 \%$, which are insignificant as error bars on this scale and gives confidence in the test as a whole. The rounding effect at the top and bottom interfaces of the column was expected due to the convolution of the detection coil response with the intrinsic step change at both these points. The correlation between $\Delta F$ and magnetic particle density is clearly highly linear with the regression curve fit shown resulting in a correlation coefficient R > $99.98 \%$. Hence, these results show that the scanning column magnetometer is a reliable method for measuring the concentration profiles of magnetic particulate dispersions.

\section{ORIGIN OF THE SCM LINEARITY AND ITS RELATIONSHIP TO THE DETECTION COIL RESPONSE}

\section{A. Total (Integrated) Response of the Coil}

To understand how a plot of $\Delta F$ against column height directly relates to pigment concentration, it is necessary to initially consider the relationship between the tuned circuit inductance and capacitance ( $L_{0}$ and $C$ respectively) and its sample-free resonance frequency, $F_{0}$, of

$$
F_{0}=\frac{1}{2 \pi \sqrt{L_{0} C}}
$$

For a change in $L_{0}$ to $\left(L_{0}+\Delta L\right)$ and the corresponding change in frequency to $\left(F_{0}+\Delta F\right)$, combined with division of both sides by $F_{0}$, leads to

$$
1+\frac{\Delta F}{F_{0}}=\frac{1}{2 \pi F_{0} \sqrt{\left(L_{0}+\Delta L\right) C}}
$$

Substitution for $F_{0}$ by (1) on the right hand side of this expression yields an equation,

$1+\frac{\Delta F}{F_{0}}=\left(1+\frac{\Delta L}{L_{0}}\right)^{-1 / 2}$.

From the small change $\Delta F / F_{0}$ of order $0.1 \%$ typically observed here, the expression may be simplified using a truncated binomial expansion to give

$\Delta F=-\frac{F_{0}}{2 L_{0}} \Delta L$

The negative sign shows an increase in inductance causes a drop in frequency as expected from (1).

With the relationship between the shift in frequency and change in induction established, the effects on the detection coil induction brought about by introducing magnetic material into its core may now be investigated. For a single turn coil carrying a current, $I$, and generating a magnetic flux, $\Phi$, its inductance is defined by $L=\Phi / I$, which by comparison with the flux density, $B=\Phi / A$, within the coil-core cross sectional area, $A$, gives

$$
L=B \frac{A}{I}
$$

Assuming a small coil field, $H$, the magnetisation curve, $M(H)$, of the material in the core is linear so that $M=\chi_{i} H$ where $\chi_{i}$ is the pigment's initial susceptibility. Now the flux density may also be described by its definition of $B=\mu_{0}(H+M)$, where $\mu_{0}$ is the permeability of free space, and can therefore be written in terms of this initial susceptibility to give $B=\mu_{0} H\left(1+\chi_{i}\right)$. Substitution for $B$ in (3) yields

$L=\left[B_{0}\left(1+\chi_{i}\right)\right] \frac{A}{I}$,

where $B_{0}=\mu_{0} H$ is the flux density when there is no material other than air in the core. By consideration of (3) again, part of the right hand side of (4) may be written in terms of the sample-free induction $L_{0}=B_{0}(A / I)$, so that (4) can now be 
(c) 2013 IEEE. Personal use of this material is permitted. Permission from IEEE must be obtained for all other users, including reprinting/ republishing this material for advertising or promotional purposes, creating new collective works for resale or redistribution to servers or lists, or reuse of any copyrighted components of this work in other works.

expressed as $L=L_{0}\left(1+\chi_{i}\right)$. Hence, any change in susceptibility due to the pigment produces a change in inductance of

$$
\Delta L=L_{0} \Delta \chi_{i}
$$

Finally, the effects of particle concentration on the dispersion's susceptibility can now be considered. In the case of the ferrimagnetic pigment used here in conjunction with the small coil field of $<0.3 \mathrm{Oe}$, it is reasonable to expect that $\Delta \chi \propto \Delta(P V C)$ provided that the concentration does not become large enough to cause nearest neighbour interactions. In the case of a suspension of superparamagnetic nanoparticles, it has been shown [7] that for a system of median diameter, $D_{V}$, then the initial susceptibility is given by

$$
\chi_{i}=\frac{(P V C) I_{s}^{\prime 2} \pi D_{V}^{3}}{18 k T} \int_{0}^{\infty} y^{3} f(y) d y
$$

where $I_{s}^{\prime}$ is the bulk material saturation magnetisation, $k$ Boltzmann's constant and $T$ the temperature. Also, since $y$ is the reduced particle diameter $D / D_{V}$ and $f(y) d y$ is the fraction of total particle volume having diameters between $y$ and $y+d y$, it can be seen that the integral is directly proportional to the system's total magnetic volume. Hence, consideration of the other system constant, $I_{s}^{\prime}$, means that at a temperature, $T$,

$$
\chi_{i} \propto(P V C) D_{V}^{3}
$$

and hence, providing that there is no on-going particle agglomeration which may vary the effective diameters of the particles (and with it the assembly's median diameter), then

$$
\Delta \chi_{i} \propto \Delta(P V C)
$$

is valid for this system as well. Consideration of (2), (5) and (6) shows that $\Delta F \propto \Delta L \propto \Delta \chi_{I} \propto \Delta(P V C)$, which means that

$$
\Delta F \propto \Delta(P V C)
$$

and thus, within the limits of a small coil field and with no ongoing particle agglomeration, we can understand theoretically how a plot of frequency shift (with respect to the sample free value) as a function of magnetic particle concentration produces the linear response observed experimentally.

\section{B. Coil Design and its Effect on the SCM Response}

The design of the detection coil is dependent upon a number of factors. In this case the SCM was intended for use in sedimentation studies and so the coil diameter needed to be maximized in order to reduce wall effects. On the other hand, the coil's response over the height of the magnetic fluid needed to be minimised if a high spatial resolution was to be achieved. This may be done by reducing the coil length, or the diameter, or by a combination of both. So, the coil needed to be as narrow as possible and have a core radius that was a trade-off between the opposing wall and resolution effects. The detection coil geometry for the UCLan SCM is shown in Fig. 3.

\section{FIG. 3 HERE}

As the radius $(R=8.5 \mathrm{~mm})$ is greater than the length $(h=2$ $\mathrm{mm}$ ) it can be seen that, for reasons of clarity, the diagram is not to scale. For the convolved SCM output to be of the pigment only would require a coil response described by a delta function. Similarly, the coil response can in itself be characterised by using a delta function approximation. This is shown in part (a) by a very thin magnetic disk $(\Delta \mathrm{z} \rightarrow 0)$ of radius $R$ being driven down the central core axis.

\section{Predicting and Measuring the Detection Coil Response Function}

As with any magnetic material, the disk can be represented by a coil with a self-inductance, $L_{D}$, carrying a current of $I_{D}$. This is shown in the equivalent circuit of the coil-material system in part (b) of Fig. 3. By virtue of the flux, $\Phi_{0}$, of the main coil that threads the $N_{D}$ turns of the disk coil, there is a mutual inductance, $L_{m}$, such that $\Phi_{0}=L_{m} I_{0}$. Likewise, by applying standard reciprocity theory (e.g. [8]) to the reciprocal linkage of $\Phi_{D}=L_{m} I_{D}$, it can be shown that for an element of the disk

$$
d \Phi_{D}=\mu_{0} M_{z} \frac{H_{z}}{I_{0}} d V
$$

where $H_{z}$ is the $z$ component of the main coil field at a magnetised disk element $M_{z}$ of volume $d V$. Integration over the total volume of the disk, written in terms of the cylindrical polar co-ordinates of Fig. 3 yields the total disk flux that threads the main coil of

$\Phi_{D}=\frac{\mu_{0}}{I_{0}} \int_{0}^{2 \pi z+\delta} \int_{z}^{R} \int_{0}^{R} M_{z} H_{z} r d r d z d \theta$

Assuming the disk material is homogenous allows the magnetisation to be written in terms of a constant susceptibility $M=\chi_{i} H$ for small $H$. Also, by symmetry, the field is constant when rotating the $d r d z$ plane around $\theta$ and so

$\Phi_{D}=2 \pi \mu_{0} \frac{\chi_{i}}{I_{0}} \int_{z}^{z+\delta} \int_{0}^{R} H_{z}^{2} r d r d z$

which means that the linkage in the main coil varies as $r H_{z}^{2}(r, z)$. By the principle of reciprocity, $\Phi_{0}=\Phi_{D}$ and the situation is that as depicted in Fig. 3 (b). This means that the coil-disk $L_{m}$ response can now be characterised by mapping out the integral of $r \mathrm{H}_{z}^{2}$ of the detection coil (over the volume of the disk) at different heights. The resultant theoretical curve is shown in the main plot of Fig. 4 and was calculated with the 
(C) 2013 IEEE. Personal use of this material is permitted. Permission from IEEE must be obtained for all other users, including reprinting/ republishing this material for advertising or promotional purposes, creating new collective works for resale or redistribution to servers or lists, or reuse of any copyrighted components of this work in other works.

aid of the Gemini numerical software package.

\section{FIG. 4 HERE}

This enables the field (in terms of flux density, $\mu_{0} H$ ) that crosses the $1 \mathrm{D}$ radius plane to be determined and means that, as the disk thickness is effectively zero, then the integral of (7) is just over $r$. Hence, each point on the main theoretical curve was found from the area under each $r B^{2}(r)$ curve at different $z$. Four of these points are shown in the inset. The radius set in the 'virtual' coil reflects the inner diameter $\sim 11.2 \mathrm{~mm}$ typically found in the experimental tubes used to hold a column of magnetic fluid.

The theory was tested experimentally by stepping a thin ferrite disk through the SCM. The disk was produced by carefully pouring a standard $\gamma-\mathrm{Fe}_{2} \mathrm{O}_{3}$ dispersion onto a PET (polyethylene terephthalate) film base so that it naturally stabilised and dried out at room temperature in a circular shape, with a disk cut out at the radius of the experimental tubes. The use of a particulate disk matches that of the fluid and ensures that the result is not affected by any eddy currents that may be induced in a continuous foil disk. The data points of this experimental measurement are shown in the main plot of Fig. 4, where it can be seen that it compares well with that of the theoretical curve to give a spatial resolution of \pm 3.4 $\mathrm{mm}$, estimated from the full width at half maximum points, and thus gives confidence in the theory providing an accurate prediction of the detection coil response.

A theory has been developed, assuming a small detection coil field and no on-going particle agglomeration, which describes the observed linearity in magnetic fluid concentration magnetometry. From consideration of the mutual detection-coil and magnetic-fluid inductance, further theoretical development to describe the instrument's response function shows excellent agreement with experiment and thus allows the response to be predicted from coil design alone.

\section{REFERENCES}

[1] J. R. Lin, Tribol. Int., vol. 49, pp. 110-115, May 2012.

[2] J. Cherubini et al., IEEE Trans. Magn., vol. 47. pp. 137-147, Jan. 2011.

[3] T. Sen et al., RSC Adv., vol. 2. pp. 5221-5228, Mar. 2012.

[4] T. Mercer and P. R. Bissell, Eur. Phys. J. Web of Conferences,.vol. 40, pp. 01003p1-01003p4, Jan. 2013.

[5] R. Zboril, M. Mashlan and D. Petridis, Chem. Mater., vol. 14 pp. 969982, Mar 2002.

[6] The International Centre for Diffraction Data, JCPDS File 24-72, http://www.icdd.com/

[7] R. W. Chantrell, J. Popplewell, and S. W. Charles, IEEE Trans. Magn., vol 14, pp. 975-977, Sep 1978 .



Fig. 1. Schematic diagram of the Scanning Column Magnetometer. The tube of magnetic dispersion is driven down through the core of a coil that forms part of an oscillatory circuit. Resultant changes in frequency are recorded over the sample height to produce a particle concentration profile
[8] B. K. Middleton, in Magnetic Recording Technology, $2^{\text {nd }}$ Ed., C. D. Mee and E. D. Daniel, Eds. New York: McGraw Hill, 1996, pp. 2.64-2.65.

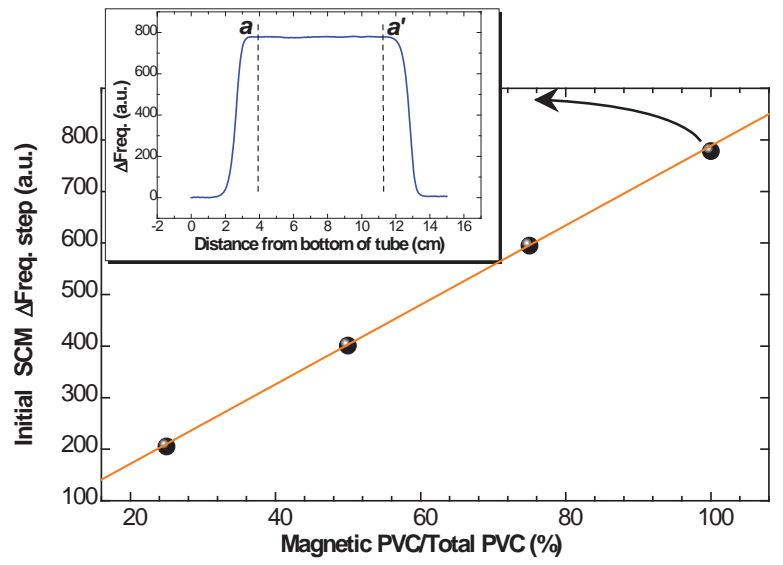

Fig. 2. Testing of the SCM output reading as a function of a suspension's magnetic pigment concentration. Each data point was found using the mean of the initial step on newly produced (homogenous) dispersions and is shown at full magnetic PVC in the inset with the calculation taken between points $a$ and $a$ '.

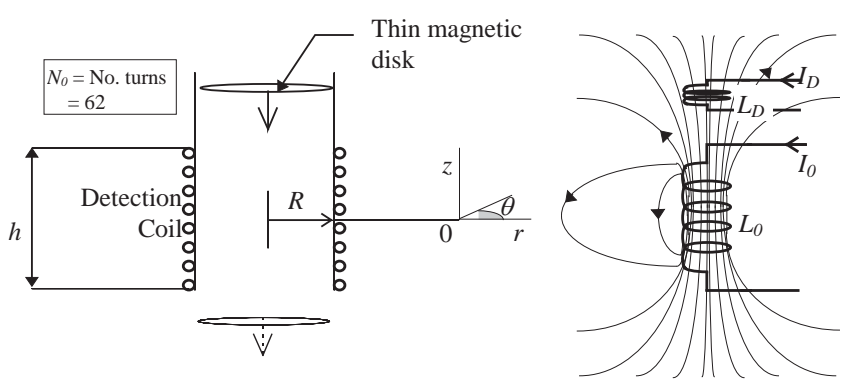

(a) Main coil design parameters

(b) Equivalent cct \& flux linkage

Fig. 3. The main coil design parameters of (a) are chosen as a trade-off between minimizing sedimentation wall effects and maximizing the spatial resolution. A delta function approximation (very thin magnetic disk) stepped through the coil core allows the coil response to be characterized by consideration of the equivalent circuit and flux linkage of part (b).

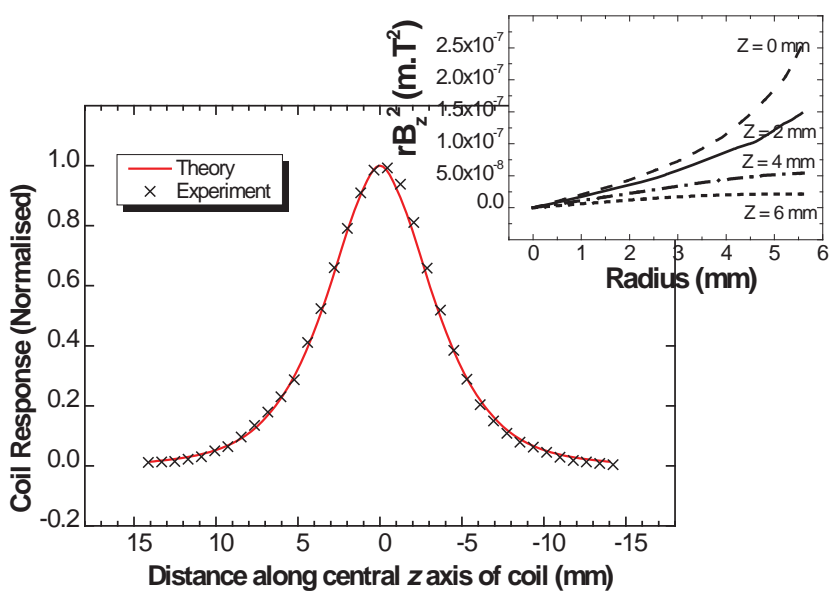

Fig. 4. Response function of the SCM. The theory curve was obtained from the integral of the coil's $r B_{z}^{2}(r)$ calculations at different points, $z$, along the coil axis with four of these curves shown in the inset. The experimental approximation to a delta function in the form of a thin ferrite disk resulted in the close fit to theory shown. 